\title{
Descarte de medicamentos e problemas ambientais: o panorama de uma comunidade no município de Fortaleza/CE
}

\author{
The disposal of medications and environmental problems: an overview of a \\ community in Fortaleza/CE
}

\author{
Alexandra de Vasconcelos Feitosa e Marisete Dantas de Aquino. \\ Universidade Federal do Ceará, Brasil \\ alexandravf@bol.com.br; marisete@ufc.br
}

\begin{abstract}
Resumo
O Brasil está entre os países que mais consomem medicamentos. Ao perderem o prazo de validade, tais medicamentos são descartados no esgoto residencial, sem nenhum cuidado, contribuindo para a geração de um passivo ambiental. A pesquisa buscou conhecer o destino final de medicamentos vencidos e descartados pela população de uma comunidade presente na cidade de Fortaleza/CE. Na pesquisa foi possível avaliar que 78,16\% dos entrevistados possuem medicamentos em casa, onde as sobras dos medicamentos são descartadas no lixo por $22,63 \%$ e $46,31 \%$ guardam para usarem outra vez. Dos entrevistados, 24,21\% relatam ter medicamentos sem a bula e 12,10\% não observam a aparência do medicamento antes de consumir. 3,95\% possuem medicamentos vencidos em casa. $68,95 \%$ acreditam o descarte de medicamentos causam problemas ambientais, sendo que 58,87\% julgam-se responsáveis pelo descarte e 5,34\% acreditam que a responsabilidade é do governo. 81,58\% relatam nunca terem recebido nenhuma informação sobre esse assunto. Com os resultados é possível afirmar que a maior parte dos usuários de medicamentos não sabe como descartar tais produtos, além de desconhecer os reais impactos ambientais provenientes do inadequado descarte e os respectivos riscos causados por ele.
\end{abstract}

Palavras-chave: Descarte; Medicamento; Meio ambiente

\begin{abstract}
Brazil is among the countries that consume more medicines. Losing the expiration date, such medications are discarded in residential sewer without any care, contributing to the generation of an environmental liability. This research sought to identify the final destination of expired and discarded medications by the population of a specific community in Fortaleza/CE. In the survey, it was possible to evaluate that $78.16 \%$ of interviewed people have medicines at home, where the remains of medicines are discarded in the trash by $22.63 \%$ and $46.31 \%$ keep them to use again. Of interviewed people, 24.21\% reported having medicines without the drug leaflet and $12.10 \%$ do not observe the appearance of the medicine before consuming. 3.95\% have expired medications at home. $68.95 \%$ believe the disposal of medicines could cause environmental problems, while $58.87 \%$ believe they are responsible for disposal and $5.34 \%$ believe it is the responsibility of the government. With the results it is possible to confirm that most medicine users do not know how to dispose of such products, in addition to ignore the real environmental impacts arising from the improper disposal and associated risks caused by it.
\end{abstract}

Keywords: Disposal; Medicine; Environment 


\section{Introdução}

Questões relacionadas à geração de resíduos resultante das atividades antrópicas vem sendo discutida nas últimas décadas, tornando-se cada vez mais foco de estudo por provocar contaminação da água, do solo, do ar e a proliferação de vetores em razão do manejo e disposição final inadequado (MOURA; SILVA, 2012).

Órgãos como a Agência Nacional de Vigilância Sanitária (ANVISA) e o Conselho Nacional do Meio Ambiente (CONAMA) têm assumido o papel de orientar, definir regras e regular a conduta dos diferentes agentes, com o intuito de preservar a saúde e o meio ambiente, garantindo a sustentabilidade (BRASIL, 2006).

Os resíduos de medicamentos são classificados por tais órgãos como resíduos químicos de classe B, compreendidos por substâncias químicas que podem apresentar riscos à saúde pública ou ao meio ambiente, dependendo de suas características de inflamabilidade, corrosividade, reatividade e toxicidade (BRASIL, 2004). Apesar de serem classificados como Resíduos de Serviço de Saúde, os resíduos de medicamento são descartados juntamente com os Resíduos Sólidos Urbanos (RSU) quando estes são gerados pela população em suas residências (VARGAS, 2014).

Por mais que as referidas legislações sejam completas e, ao mesmo tempo específicas, elas não oferecem o mesmo tipo de informações e orientações para o consumidor final, que é classificado como aquele que faz uso de medicamentos em sua residência. O descarte efetuado por esse grupo é o que apresenta maior lacuna na legislação, uma vez que não há ordenamento jurídico brasileiro, normas e regulamentos específicos no que se refere ao manejo e gerenciamento desses resíduos (BOER; FERNANDES, 2011).

Em agosto de 2010, o congresso Nacional promulgou a Lei $N^{\circ} 12.305 / 10$, instituindo a Política Nacional de Resíduos Sólidos (PNRS) que sugere a responsabilidade pela coleta, tratamento e destinação final seja compartilhada na questão dos resíduos sólidos (BRASIL, 2010; BALBINO; BALBINO, 2011; MARCHI, 2011).

Essa nova política preconiza além da gestão de resíduos que deverão incluir o gerenciamento de resíduos perigosos domiciliares, incluindo os de medicamentos, a coleta seletiva e os sistemas de logística reversa, além disso, ela orienta a redução da quantidade de resíduos a serem dispostos. Assim, a proposição das ações em busca de soluções para uma gestão ambientalmente correta dos resíduos, tem como objetivos fundamentais e norteadores, em ordem de prioridade: a não geração, redução, reutilização, reciclagem e a destinação ambientalmente adequada dos rejeitos (BRASIL, 2010; VARGAS, 2014).

Os fármacos têm recebido atenção especial por ser considerado um poluente emergente, devido a sua utilização em grandes quantidades na medicina humana e veterinária. A facilidade de aquisição desses medicamentos e o incentivo dos meios de comunicação geram um uso excessivo e consequentemente um acúmulo de tais produtos nas residências, o que provoca um problema de saúde pública como também de ordem ambiental, sendo necessária a discussão sobre o gerenciamento de medicamentos em desuso e propor soluções para minimizar os problemas gerados por eles (JOÃ̃, 2011).

Diferentes partes do mundo têm identificado à presença de fármacos tanto nas águas, como no solo. Fármacos são compostos bastante persistentes e pouco biodegradáveis e quando vencidos ou descartados indevidamente são lançados diretamente nas pias e vasos sanitários, chegam as Estações de Tratamento de Esgoto (ETE) na sua forma original, sem sofrer alterações do metabolismo no corpo humano, contribuindo de forma mais acentuada para a contaminação ambiental (ZUCCATO et al., 2006; CARVALHO et al., 2009; EICKHOFF et al., 2009), sendo a porcentagem de remoção desses compostos nas ETEs bastante baixa (ZAPPAROLI et al., 2011).

A forma mais importante de entrada destas substâncias no sistema aquático é por meio de efluentes de indústrias farmacêuticas, descartes de medicamentos com prazos de validade vencidos, efluentes hospitalares, entre outros (ZHANG et al., 2010).

Os fármacos biologicamente ativos presentes no ambiente interagem com a biota do meio interferindo significativamente na fisiologia, no metabolismo e no comportamento das espécies, como exemplo a feminização de peixes machos presentes em rios contaminados com descarte de efluentes de Estações de Tratamento de Esgoto. Alguns causam efeitos secundários como a alteração na defesa imunológica de organismos tornando-os mais suscetíveis a doenças (BILA; DEZOTTI, 2003; REIS FILHO et al., 2007).

Dentre os fármacos de importância ambiental devido a sua toxicidade, persistência no meio ambiente e quantidades consumidas estão os beta-bloqueadores, analgésicos, hormônios esteroides, antinflamatórios e medicação para o tratamento de câncer, compostos neuro-ativos, agentes redutores de lipídeos no sangue, antiparasitas e antibióticos (BILA; DEZOTTI, 2003; CARVALHO et al., 2009; EICKHOFF et al., 2009).

Inúmeras são as causas das sobras de medicamento, tais como: quantidade maior que a posologia, estímulo à aquisição não necessária através de propagandas, a não adesão dos pacientes ao tratamento prescrito entre outros (MENDES et al., 2010; ALVARENGA; NICOLETTI, 2010).

O descarte impróprio desses produtos deve ser estudado, uma vez que eles contaminam o solo e a água de forma a comprometer direta e indiretamente as propriedades naturais. Assim, torna-se necessária a discussão sobre o gerenciamento de fármacos em desuso e propor soluções para minimizar os problemas gerados por eles (JOÃ̃, 2011).

Tendo em vista a realidade do consumo de medicamentos no país, o panorama de destinação final dos mesmos, as consequências ao meio ambiente e a saúde da população, este trabalho tem como objetivo verificar a 
contextualização atual do desperdício e descarte aleatório em desuso, vencidos ou sobras de medicamentos pela população do bairro José Walter, situado no Município de Fortaleza/CE e a visão dos mesmos em relação aos prejuízos causados à população e ao meio ambiente que esses produtos podem gerar.

\section{Metodologia}

O estudo foi do tipo exploratório-explicativo, com abordagem quantitativa. A pesquisa foi realizada em um bairro na cidade de Fortaleza/CE denominado José Walter, que segundo dados da prefeitura de Fortaleza a população é de 33.427 habitantes sendo formado por 13,07 Km2 de extensão, cujo IDH da população é de 0,51 (Fortaleza, 2015).

Para a coleta de dados foi utilizado questionário padronizado, contendo questões de fácil compreensão, previamente testado, baseado no trabalho publicado por Gasparini e colaboradores (2011), sendo composto por duas etapas. A primeira formada por seis questões objetivas relacionadas aos dados sócios demográficos e, a segunda, com questões objetivas e subjetivas referentes aos fármacos e seu descarte.

Com o intuito de avaliar uma amostra significativa dessa população, foi utilizada a Equação 1 para determinação do tamanho da amostra () com base na estimativa da média populacional. Foi considerado um erro amostral de $5 \%$ e o intervalo de confiança adotado foi de $95 \%$.

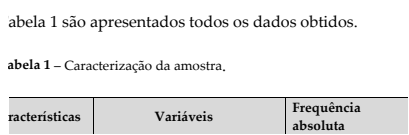

Onde:

$=$ tamanho da amostra

$\mathrm{Z}=$ abscissa da distribuição normal padrão

$\mathrm{p}=$ estimativa da proporção da característica pesquisada no universo; $\mathrm{q}=1-\mathrm{p}$

$\mathrm{N}=$ tamanho da população

$\mathrm{d}=$ erro amostral

A pesquisa foi realizada com 380 participantes, selecionados aleatoriamente, residentes no bairro. Foram incluídos no estudo usuários de ambos os sexos, maiores de 18 anos, sem distinções quanto à etnia, grau de escolaridade, níveis de renda ou estado civil. Esse espectro de amostra populacional atende aos critérios e necessidades estatísticas.

A participação na pesquisa teve caráter voluntário e os respondentes foram informados previamente dos objetivos da pesquisa, da natureza sigilosa das informações, do resguardo do anonimato e da possibilidade de desistência do preenchimento do questionário a qualquer tempo caso desejassem.

O desenvolvimento da pesquisa tem o intuito de verificar o perfil sócio-econômico dos usuários de me- dicamentos residentes no bairro José Walter (FortalezaCE), avaliar o comportamento das pessoas ao adquirir e utilizar os medicamentos, além de traçar o panorama da consciência e consequências sobre o descarte dos medicamentos.

\section{Resultados e discussões}

\section{Análise do perfil sócio-demográfico}

Com a pesquisa observou que a população é composta, por mais jovens que idosos, pois a população idosa entrevistada foi de $2,89 \%$, sendo a faixa etária predominante de 18 a 25 anos (52,63\%). O gênero predominante foi o feminino com $55,26 \%$ e $44,74 \%$ os pesquisados do gênero masculino. Em relação ao nível de escolaridade da população residente no bairro, observa-se que a escolaridade predominante é o ensino médio incompleto $(45,79 \%)$ e completo $(17,89 \%)$, no entanto, o bairro possui uma quantidade pequena de analfabetos. Os dados obtidos em função da renda foram: baixa 41,58\%, renda média 55,53\% e alta 2,79\%. Na Tabela 1 são apresentados todos os dados obtidos.

Inicialmente foi verificada a presença de medicamentos nas residências pesquisadas e foi constatado que $78,16 \%$ dos entrevistados possuem esses produtos em casa e apenas $21,84 \%$ não possuem. Assim, a pesquisa mostrou a existência de um estoque domiciliar, também conhecida como farmácia caseira. A facilidade de aquisição de medicamentos poder ser a causa da elevada porcentagem de pessoas que possuem medicamentos em casa, uma vez que muitos medicamentos de uso comum como os analgésicos e antitérmicos estão disponíveis em farmácias, drogarias e supermercados, podendo ser obtidos sem a necessidade de receita médica (AUTOMEDICAÇÃO, 2001).

A pesquisa também avaliou a forma de acondicionamento dos medicamentos nas residências, uma vez que fatores como temperatura, presença de oxigênio, luz solar, radiação e umidade podem modificar o teor do fármaco (BUENO et al., 2009). É de grande importância que a farmácia caseira possa garantir a qualidade dos medicamentos, através do adequado armazenamento destes,

Os parâmetros abordados foram na pesquisa foram: Presença de bula;

Facilidade de acesso das crianças aos medicamentos;

Observância do aspecto e data de validade dos medicamentos antes do uso.

Em relação à presença de bula com o respectivo medicamento foi constatado que 51,05\% dos entrevistados guardam todos os medicamentos com a bula, 24,21\% não a possuem e 24,74\% possuem somente algumas.

A importância da bula é que ela descreve, de forma mais detalhada, as informações necessárias para a utilização mais segura do produto pelo paciente (ANVISA, 
Tabela 1 - Caracterização da amostra.

\begin{tabular}{|c|c|c|c|}
\hline Características & Variáveis & Frequência absoluta & Frequência (\%) \\
\hline \multirow{6}{*}{ Faixa etária } & 18 a 25 & 200 & 52,63 \\
\hline & 26 a 35 & 56 & 14,74 \\
\hline & 36 a 50 & 82 & 21,58 \\
\hline & 51 a 65 & 31 & 8,16 \\
\hline & $>65$ & 11 & 2,89 \\
\hline & Total & 380 & 100 \\
\hline \multirow{3}{*}{ Gênero } & Masculino & 170 & 44,74 \\
\hline & Feminino & 210 & 55,26 \\
\hline & Total & 380 & 100 \\
\hline \multirow{8}{*}{ Escolaridade } & Analfabeto & 14 & 3,68 \\
\hline & $\begin{array}{c}\text { Ensino fundamental } \\
\text { incompleto }\end{array}$ & 46 & 12,10 \\
\hline & $\begin{array}{l}\text { Ensino fundamental } \\
\text { completo }\end{array}$ & 30 & 7,89 \\
\hline & Ensino médio incompleto & 174 & 45,79 \\
\hline & Ensino médio completo & 68 & 17,89 \\
\hline & Ensino superior incompleto & 10 & 2,63 \\
\hline & Ensino superior completo & 38 & 10 \\
\hline & Total & 380 & 100 \\
\hline \multirow{4}{*}{ Renda Mensal } & Baixa & 158 & 41,58 \\
\hline & Média & 211 & 55,53 \\
\hline & Alta & 11 & 2,79 \\
\hline & Total & 380 & 100 \\
\hline
\end{tabular}

2010). O resultado mostra que grande parte da população residente nesse bairro não reconhece a importância da bula como fonte de informação escrita, pois nela são fornecidas informações sobre ação esperada do medicamento, contra-indicações, ação esperada do medicamento, prazo de validade, cuidados de administração, interrupção do tratamento, reações adversas, Ingestão concomitante com outras substâncias e contra-indicações.

Os dados também apontam que $89,47 \%$ dos participantes não deixam os medicamentos ao alcance de crianças, mas ainda existe uma minoria (10,53\%) que admitem o descuido de deixar tais produtos ao alcance das crianças. Este resultado mostra que elevada porcentagem da população tem consciência de que os medicamentos oferecem riscos as crianças e que devem ficar longe delas, evitando-se possíveis acidentes.

De acordo com dados do Sistema Nacional de Informações Tóxico-farmacológicas da Fundação Oswaldo Cruz, foram registrados no Brasil mais de 80 mil casos de intoxicações e, em quase $25 \%$ das notificações, as vítimas tinham até 5 anos. Os medicamentos estão em primeiro lugar no ranking de intoxicação e entre os principais agentes tóxicos destacam-se os medicamentos (35\%), os domissanitários (18\%) e os produtos químicos industriais (9\%) (BRASIL, 2014). Essas intoxicações são decorrentes de situações facilitadoras, das características peculiares às fases de desenvolvimento da criança e do pouco incentivo às medidas preventivas (LOURENÇO et al., 2008; SIQUEIRA et al., 2008).

Observar o aspecto do medicamento é muito significante para garantir que este, não esteja em degradação visível (FANHANI et al. 2006). Os participantes da pesquisa foram questionados sobre o costume de verificação do aspecto e do prazo de validade dos medicamentos antes do consumo e foi constatado que $87,90 \%$, afirmou verificar a aparência e prazo de validade dos medicamentos antes do consumo e 12,10\% não possui esse hábito. Durante a aplicação do questionário percebeu-se que as pessoas estão mais conscientes da importância de se verificar o prazo de validade dos medicamentos.

A Tabela 2 apresenta os resultados da pesquisa em relação ao comportamento na aquisição, acondiciona- 
Tabela 2 - Características dos entrevistados quanto aos hábitos de consumo de medicamentos.

\begin{tabular}{c|c|c}
\hline Variáveis & Frequência absoluta & Frequência (\%) \\
\hline \multicolumn{3}{c}{ Possui medicamentos em casa? } \\
\hline Sim & 297 & 78,16 \\
\hline Não & 83 & 21,84 \\
\hline Há presença de bula com o respectivo medicamento? \\
\hline Sim & 194 & 51,05 \\
\hline Não & 92 & 24,21 \\
\hline Somente algumas & 94 & 24,74 \\
\hline \multicolumn{3}{c}{ Os medicamentos estão ao alcance de crianças? } \\
\hline Sim & 40 & 10,53 \\
\hline Não & 340 & 89,47 \\
\hline Observa aparência e a data de validade do medicamento antes de utilizá-lo? \\
\hline Sim & 334 & 87,90 \\
\hline Não & 46 & 12,10 \\
\hline
\end{tabular}

mento e uso do medicamento dos entrevistados.

\section{Consciência da população sobre o descarte de}

\section{medicamentos}

Para verificar o comportamento dos moradores em relação com o procedimento adotado com as sobras dos medicamentos foi constatado que $9,74 \%$ devolvem à unidade ou ao agente de saúde, $46,31 \%$ guarda para usar outra vez, $22,63 \%$ põe no lixo, $4,74 \%$ doam aos vizinhos, amigos e/ou parentes e $16,58 \%$ afirmam que não há sobra de medicamento após o uso.

A Figura 1 apresenta o destino dado às sobras dos medicamentos após a conclusão do tratamento, com o resultado obtido, pode-se dizer que a farmácia caseira promove a automedicação. Outro problema que pode gerar é o uso de medicamentos inadequados para a patologia existente, quando sintomas semelhantes são causados por patologias distintas. Também pode facilitar trocas e, quando o medicamento estiver vencido, causar danos a saúde ou a não observância do efeito esperado (BUENO et al., 2009).

Também foi constatada na pesquisa que uma pequena parcela dos entrevistados possui medicamentos vencidos $(3,95 \%)$ em suas residências. No entanto, esses medicamentos são descartados no lixo comum (Tabela 3). Desta forma são levados a aterros sanitários comuns, não recebendo o tratamento adequado de incineração e assim, contaminando o meio ambiente pelos resíduos químicos que os compõe (HOPPE; ARAÚJO, 2012).

Com o estudo, observa-se que as informações à população sobre o descarte e armazenamento de medicamentos é um assunto muito distante da realidade. $81,58 \%$ dos entrevistados afirmam que nunca foram orientados sobre o descarte e armazenamento de medi- camento, o que difere em 18,42\% asseguram ter recebido tais informações.

Os dados apontam que a principal forma de descarte dos medicamentos vencidos é o lixo comum (73,68\%), em seguida aparece o descarte no vaso sanitário com $13,95 \%$, na pia com $11,31 \%$, além de outras formas como no hospital com $0,79 \%$ e $0,27 \%$ entrega ao agente de saúde. A distribuição da forma de descarte é apresentada na Figura 2.

Apesar de não ser do conhecimento da maioria da população, o lixo comum, vaso sanitário ou a pia não são os destinos corretos para eliminação desses produtos. Esse descarte aleatório, tanto no lixo comum como na rede pública de esgoto pode trazer como consequências a contaminação da água, do solo e de animais (RIBEIRO; BINSFELD, 2013), desta forma, há a necessidade de conscientização com a população sobre os riscos e a destinação correta de tais resíduos.

No estudo foi investigado o nível de consciência que os participantes da pesquisa possuem em relação aos prejuízos que podem trazem o descarte incorreto desses produtos. O resultado obtido é que $71,58 \%$ dos entrevistados acreditam que os descartes incorretos trazem prejuízos à população. No entanto, $48,16 \%$ não sabem quais são esses prejuízos. Outras respostas mais relevantes foram: $20,59 \%$ consideram que o descarte incorreto polui o meio ambiente, $18,01 \%$ afirmam que causam doenças, no entanto, não relataram quais doenças e 5,15\% afirmam que causam envenenamento. Os resultados são apresentados na Tabela 3 .

A partir destes resultados é possível afirmar que a maioria da população avaliada não detém a devida proporção de consciência uma vez que uma grande parte dos indivíduos que afirmavam ter o descarte incorreto proporciona prejuízos à população, 48,16\% não sabem que prejuízos são esses. 


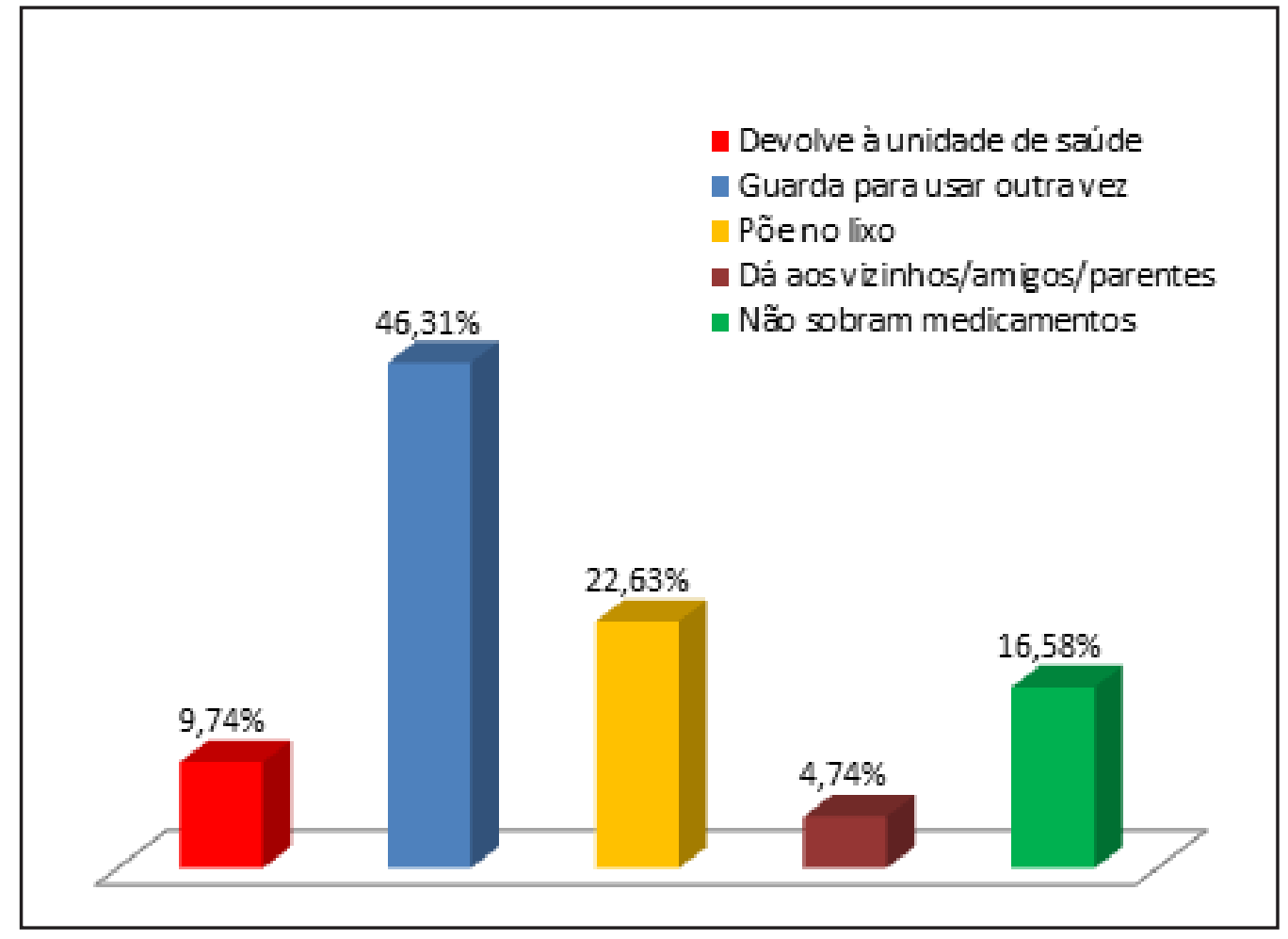

Figura 1 - Destino das sobras de medicamentos realizadas pela população em estudo.

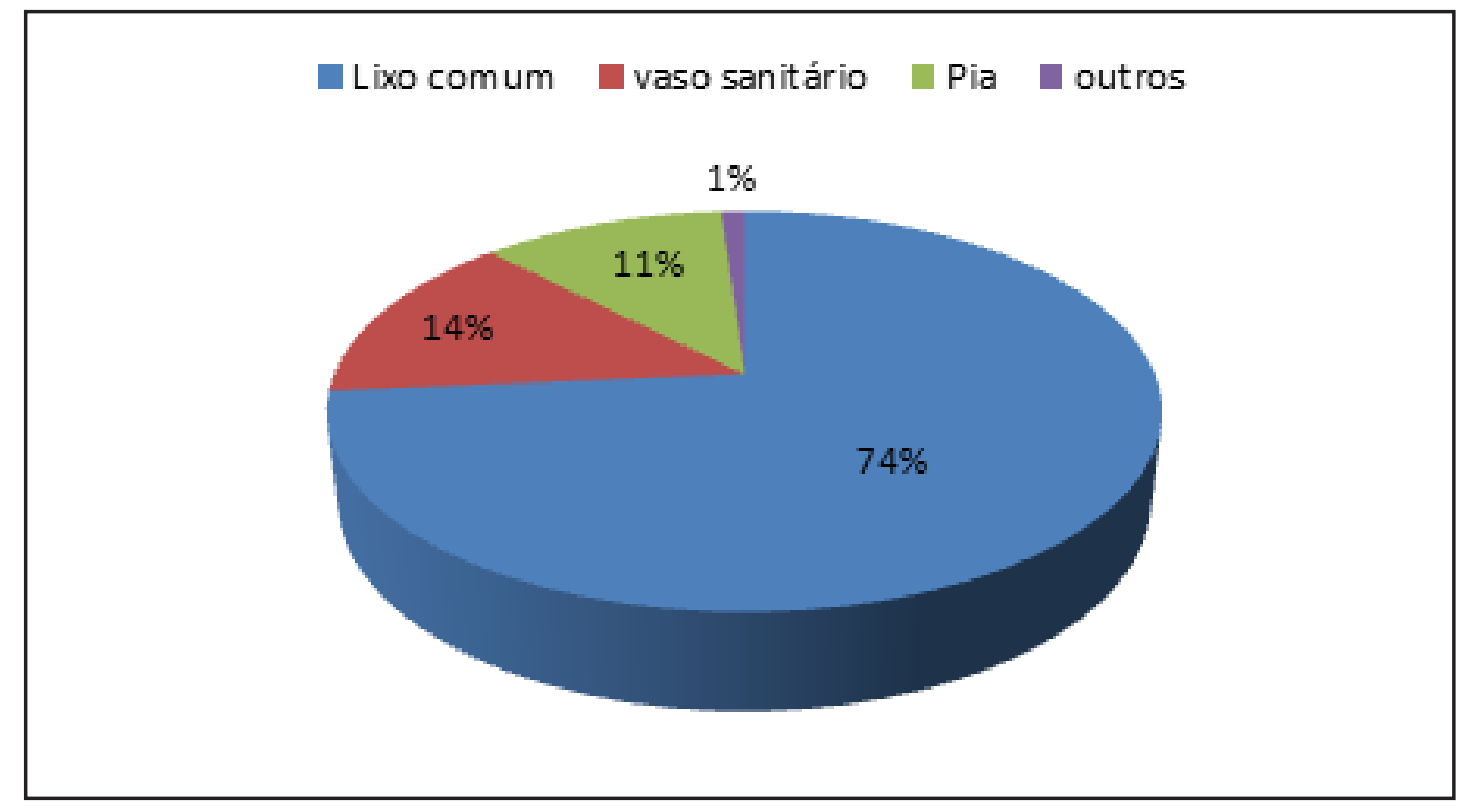

Figura 2 - Distribuição da forma de descarte de medicamentos vencidos. 
Tabela 3 - Distribuição da presença de medicamentos vencidos nos domicílios, consciência dos prejuízos ocorridos pelo descarte incorreto de medicamentos.

\begin{tabular}{c|c|c}
\hline Variável & Frequência & Porcentagem \\
\hline \multicolumn{2}{c}{ Você possui medicamentos vencidos em sua residência? } \\
\hline Sim & 15 & 3,95 \\
\hline Não & 365 & 96,05 \\
\hline Se sim, o que faz com eles? & 15 & 100 \\
\hline Descarta no lixo comum &
\end{tabular}

Você já recebeu alguma informação quanto ao armazenamento e descarte de medicamento?

\begin{tabular}{c|c|c}
\hline Sim & 311 & $18,16 \%$ \\
\hline Não & 69 & $81,84 \%$ \\
\hline
\end{tabular}

Você considera que o descarte incorreto de medicamento traz algum prejuízo para a população?

\begin{tabular}{c|c|c}
\hline Sim & 272 & 71,58 \\
\hline Não & 108 & 28,42 \\
\hline \multicolumn{2}{|c|}{ Se sim, qual? } \\
\hline Doenças & 49 & 18,01 \\
\hline Contaminação do solo ou água & 4 & 1,47 \\
\hline Poluição & 56 & 20,59 \\
\hline Envenenamento & 14 & 5,15 \\
\hline Desperdício de medicamentos & 9 & 3,31 \\
\hline Intoxicação/alergia & 1 & 0,37 \\
\hline Uso inadequado do medicamento & 8 & 2,94 \\
\hline Não sabe responder & 131 & 48,16
\end{tabular}

Assim, deve-se reforçar o fato de que o descarte correto de medicamentos enfrenta vários entraves, como a falta de conhecimento da população acerca do assunto e de informação, além de fiscalização a respeito do destino dado a esses produtos e também a ausência de qualquer orientação por parte do fabricante, tanto na embalagem quanto na bula do fármaco (TESSEROLLI et al., 2013).

\section{Avaliação da consciência dos impactos ambientais}

\section{em função do descarte inadequado}

No estudo também foi avaliado à consciência ambiental da comunidade, além do questionamento dos possíveis responsáveis pelo descarte de medicamentos e os impactos ambientais causados por esses produtos. Os resultados são apresentados na Tabela 4.
$68,95 \%$ acreditam que o descarte incorreto ocasione prejuízos ao meio ambiente e dessa amostra $56,87 \%$ consideram que a própria população é responsável por essa atitude. Segundo os entrevistados, além da população, eles acreditam que o governo, a Secretaria de Saúde, a indústria farmacêutica, o agente de saúde e a vigilância sanitária também são responsáveis pelo descarte dos medicamentos. No entanto, $33,97 \%$ dos entrevistados não sabem responder tal questionamento.

Quando foi questionado qual impacto ambiental o descarte de medicamento provocava foi obtido o seguinte resultado: $15,65 \%$ acreditam que a poluição seja o impacto causado por esses produtos; $5,72 \%$ responderam contaminação do solo e 2,29\% contaminação da água. Também foram obtidas algumas respostas que mostra a falta de informação da população frente a essa temática, além de respostas muito vagas como: doenças 
(4,02\%), efeito estufa (2,29\%), além de 69,08\% não sabem responder ao questionamento.

Estudos apontam que fármacos de diferentes classes terapêuticas, têm sido detectados em esgoto doméstico, águas superficiais e subterrâneas em concentrações na faixa de $\mathrm{ng} / \mathrm{L}$ a $\mu \mathrm{g} / \mathrm{L}$ em várias partes do mundo (GROVER et al., 2011; XU et al., 2012; REH et al., 2013; FANG et al., 2012; QUEIROZ et al., 2011). Em sistemas aquáticos, os antibióticos, antineoplásicos, hormônios sexuais são extremamente tóxicos para organismos vivos como algas e peixes (SANDESON et al., 2004).

Os efeitos deletérios que a presença desses compostos pode provocar são: o desequilíbrio da ecologia microbiana, a proliferação de agentes patogênicos resistentes, além de efeitos inespecíficos na saúde humana, destacando a ocorrência de processos alérgicos, o que representa um alerta para a necessidade de se desenvolver tecnologias de tratamento adequadas para a remoção de tais compostos (ZAPPAROLI et al., 2011).

A partir deste cenário, existe a necessidade da preocupação relacionada com o modo correto de descarte de medicamentos, uma vez que não há pontos de coleta para medicamentos em vigor no país. O órgão responsável pela regulamentação dos meios de descarte desses me- dicamentos é a Agência Nacional de Vigilância Sanitária (ANVISA, 2010), que através da resolução RDC 306/04 (Brasil, 2004), exige que estabelecimentos de serviços de saúde disponham de Plano de Gerenciamento de Resíduos de Serviço de Saúde (PGRSS) e a resolução 358 do Conselho Nacional do Meio Ambiente (CONAMA) (Brasil, 2005), dispõe sobre o tratamento e disposição final dos resíduos de serviços de saúde, entre outros.

Através da Lei Federal N¹2.305 de 2010, prevista na Política Nacional de Resíduos Sólidos (PNRS), farmácias, postos de saúde e laboratórios devem receber medicamentos vencidos e destiná-los de forma ambientalmente correta, evitando o que poderia ser um foco gerador de contaminação e poluição.

De acordo com a legislação brasileira, os serviços de saúde são responsáveis pelo gerenciamento de todos os resíduos dos serviços de saúde por eles gerados, devendo atender às normas e exigências legais, desde o momento de sua geração até a destinação final (BRASIL, 2006). No entanto, a legislação existente não obriga as farmácias a fazerem o descarte dos medicamentos vencidos na mão do cliente, como também permite ao consumidor descartar os medicamentos no lixo comum, em pias ou vasos sanitários, de onde vão para os esgotos (TESSARO;

Tabela 4 - Distribuição da consciência ambiental da comunidade avaliada, dos possíveis responsáveis pelo descarte de medicamentos os impactos ambientais causados por esses produtos.

\begin{tabular}{c|c|c}
\hline Variável & Frequência & Porcentagem (\%) \\
\hline Você acredita que o descarte incorreto de medicamentos traz problemas ambientais? \\
\hline Sim & 262 & 68,95 \\
\hline Não & 118 & 31,05 \\
\hline \multicolumn{1}{c|}{ Se sim, quem é o responsável? } \\
\hline Sociedade & 149 & 56,87 \\
\hline Governo & 14 & 5,34 \\
\hline Secretaria de Saúde & 5 & 1,90 \\
\hline Indústria farmacêutica & 3 & 1,14 \\
\hline Agente de saúde & 1 & 0,38 \\
\hline Vigilância sanitária & 1 & 0,38 \\
\hline Não sabe responder & 89 & 33,97 \\
\hline Se sim, qual o impacto ambiental que você conhece? \\
\hline Doenças & 11 & 4,20 \\
\hline Danos ao meio ambiente & 2 & 0,76 \\
\hline Efeito estufa & 6 & 2,29 \\
\hline Pontuição & 41 & 15,65 \\
\hline Não sabe responder & 15 & 5,72 \\
\hline Contaminação do solo & 6 & 2,29 \\
\hline Contanão da água & 181 & 69,08 \\
\hline
\end{tabular}


ZANCANARO, 2013).

\section{Conclusões}

Com o presente trabalho, foi diagnosticado que o uso de medicamentos se faz presente de modo intenso na população estudada residente no bairro José Walter e ainda constatou-se a necessidade da orientação da população quanto ao uso racional destes medicamentos.

O problema de resíduos farmacológicos liberados no meio ambiente é muito complexo e a contaminação dos recursos ambientais por estes compostos descartados de forma incorreta pode gerar um grande impacto ambiental.

Os resultados comprovam que a grande parte da população possui medicamentos em casa e o hábito de descartar os medicamentos de forma incorreta e em locais inadequados é atribuído pelo fato de não existir nenhuma orientação formal sobre o assunto por parte dos órgãos competentes em relação ao descarte correto de sobras medicamentos, além dos vencidos.

Percebe-se que os destinos mais frequentes de descarte de medicamentos são o lixo doméstico $(73,68 \%)$ e o vaso sanitário (13,95\%). A pesquisa apresentou um nível de conscientização satisfatória, pois mais da metade dos entrevistados acredita que o descarte indevido de medicamento tanto traz prejuízo à população $(71,58 \%)$ como contribuem para os problemas ambientais $(68,95 \%)$. No entanto, quando se questiona quais são esses problemas, grande parte dos entrevistados não sabe responder, além disso, as formas de descarte continuam inadequadas.

Apesar da relevante importância sobre o assunto, ainda não existe no Brasil orientação sobre os procedimentos para descarte de medicamentos vencidos administrados na própria residência, assim, esse trabalho evidenciou a necessidade de leis mais específicas e programas de conscientização de uso consciente e descarte de medicamentos, além de programas de recolhimento de tais produtos utilizando a logística reversa como ferramenta. Além disso, para amenizar os riscos de contaminação ambiental pelo descarte incorreto de medicamentos, a melhor abordagem é a minimização da geração destes resíduos, através de prescrições racionais, adequação das embalagens aos tratamentos, dispensação adequada e cumprimento das prescrições por parte dos usuários.

\section{Referências}

ANVISA. Agência Nacional de Vigilância Sanitária. O que devemos saber sobre medicamentos. 2010. Disponível a partir de: http://www.paulinia.sp.gov.br/downloads/ Cartilha_medicamentos.pdf.

ALVARENGA, L. S. V.; NICOLETTI, M. A. Descarte doméstico de medicamentos e algumas considerações sobre o impacto ambiental decorrente. Revista Saúde, v. 4, p. 34-39, 2010.

BALBINO, E. C.; BALBINO, M. L. C. O descarte de medicamentos no Brasil: Um olhar socioeconômico e ambiental do lixo farmacêutico. Âmbito Jurídico, Rio Grande, XIV, v. 86, 2011. Disponível a partir de: http:// www.ambitouridico.com.br/site/index.php?artigo id=9187\&n_link=revista_artigos_leitura.

BILA, D. M.; DEZOTTI, M. Fármacos no meio ambiente. Quim. Nova, v. 26, n. 4, p. 523-530, 2003.

BOER, N; FERNANDES, B. O. Descarte de medicamentos: um modelo de logística reversa. In: Congresso Responsabilidade e Reciprocidade, Restinga Seca, 2011.

BRASIL. Lei $\mathrm{N}^{\circ} 12.305$, de 2 de agosto de 2010. Institui a Política Nacional de Resíduos Sólidos; altera a Lei ${ }^{\circ}$ 9.605, de 12 de fevereiro de 1998; e dá outras providências. Brasília, 2010.

BRASIL. Resolução RDC N 306, de 7 de dezembro de 2004. Ministério da Saúde. Agencia Nacional de Vigilância Sanitária (ANVISA). Dispõe sobre o Regulamento técnico para o gerenciamento de resíduos dos serviços de saúde. Brasília, 2004.

BRASIL. MINISTÉRIO DA SAÚDE. Fundação Oswaldo Cruz. Sistema Nacional de Informações TóxicoFarmacológicas - SINITOX. Centro de Informação Científica e Tecnológica. Crianças são 25\% das vítimas de intoxicação. Brasil, 2005. Disponível a partir de: http:// portal.fiocruz.br/pt-br/node/1911.

BRASIL. Resolução No 358, de 29 de abril de 2005. Ministério do Meio Ambiente. Conselho Nacional do Meio Ambiente (CONAMA). Dispõe sobre o tratamento e a disposição final dos resíduos dos serviços de saúde e dá outras providências. Brasília, 2005.

BRASIL. Manual de Gerenciamento de resíduos de serviços de saúde. Ministério da Saúde. Agencia Nacional de Vigilância Sanitária (ANVISA). Brasília, 2006.

BUENO, C. S.; WEBER, D.; OLIVEIRA, K. R. Farmácia caseira e descarte de medicamentos no bairro Luiz Fogliatto do município de Ijuí - RS. Rev. Ciências Farm. Básica Apl., v. 30, n. 2, p. 75-82, 2009.

AUTOMEDICAÇÃO. Revista da Associação Médica Brasileira, São Paulo, v. 47, n.4, p. 269-270, 2001.

CARVALHO, E. V; FERREIRA, E.; SANTOS, C. Aspectos legais e toxicológicos do descarte de medicamentos. Rev. Bras. de Toxicologia, v. 22, p. 1-8, 2009. 
EICKHOFF, P.; HEINECK, I.; SEIXAS, L. J. Gerenciamento e destinação final de medicamentos: uma discussão sobre o problema. Rev. Bras. Farm., v. 90, n. 1, p. 64-68, 2009.

FANG, T.-H; NAN, F. H; CHIN, T. S.; FENG, H. M. The occurrence and distribution of pharmaceutical compounds in the effluents of a major sewage treatment plant in Northern Taiwan and the receiving coast al waters. Marine Pollution Bulletin, v. 64, p. 1435-1444, 2012.

Fanhani, H. R. et al. Avaliação domiciliar da utilização de medicamentos por moradores do Jardim Tarumã, município de Umuarama - PR. Arq. Ciênc. Saúde Unipar, v. 10, n. 3, p. 127-131, 2006.

FORTALEZA, 2015. Disponível a partir de: http://www. fortaleza.ce.gov.br/regionais/regional-V.

GASPARINI, J. C.; GASPARINI, A. R.; FRIGIERI, M. C. Estudo do descarte de medicamentos consciência ambiental no município de Catanduva-SP. Rev. Ciência \& Tecnologia, v. 2, n. 1, p. 38-51, 2011.

GROVER, D. P.; ZHOU, J. L.; FRICKERS, P. E.; READMAN, J. W. Improved removal of estrogenic and pharmaceutical compounds in sewage effluent by full scale granular activated carbon: Impact on receiving river water. Journal of Hazardous Materials, v. 185, p. 1005-1011, 2011.

HOPPE, T. R. G.; ARAÚJO, L. E. B. Contaminação do meio ambiente pelo descarte inadequado de medicamentos vencidos ou não utilizados. Rev. Monografias ambientais, v. 6, n. 6, p. 1248-1262, 2012.

JOÃO, W. S. J. Descarte de medicamentos. Pharmacia Brasileira, v. 82, n. 82, p. 14-16, 2011.

LOURENÇO, J.; FURTADO, B. M. A.; BONFIM, C. Intoxicações exógenas em crianças atendidas em uma unidade de emergência pediátrica. Acta Paul Enferm.,vol. 21, p. 282-286, 2008.

MARCHI, C. M. D. F. Cenário mundial dos resíduos sólidos e o comportamento corporativo brasileiro frente à logística reversa. Perspectivas em Gestão \& Conhecimento, João Pessoa, v. 1, n. 2, p. 118-135, 2011.

MENDES, Z.; CRISÓSTOMO, S.; MARQUES, F. B; MARTINS, A. P.; RODRIGUES, V.; RIBEIRO, C. F. Desperdício de medicamentos no ambulatório em Portugal. Rev Port Clin Geral, v. 26, p.12-20, 2010.

MOURA, L. L.; SILVA, R. F. Avaliação do impacto ambiental gerado pelos resíduos de um hospital universitário de alta complexidade. In: Simpósio de excelência em gestão e tecnologia - IX SEGeT, 2012.
QUEIROZ, F. B; BRANDT, E. M. F.; AQUINO, S. F.; CHERNICHARO, C. A. L.; AFONSO, R. J. C. F. Occurrence of pharmaceuticals and endocrine disruptors in raw sewage and their behaviour in UASB reactors operated at different hydraulic retention times. Water Science and Technology, v. 66, n. 12, p. 2562-2569, 2012.

REH, R.; LICHA, T.; GEYER, T.; NÖDLER, K.; SAUTER, $\mathrm{M}$. Occurrence and spatial distribution of organic micropollutants in a complex hydrogeological karst system during low flow and high flow periods, results of a two-year study. Science of the Total Environment, v. 443, p. 438-445, 2013.

REIS FILHO, R. W.; BARREIRO, J. C.; VIEIRA, E. M.; CASS, Q. B. Fármacos, ETEs e corpos hídricos. Revista Ambi - Água, v. 2, n. 3, p. 54-61, 2007.

RIBEIRO, M. A.; BINSFELD, P. C. Descarte de medicamentos vencidos ou não utilizados: riscos e avanços recentes. 2013. Disponível a partir de: http://www.cpgls.pucgoias.edu.br/8mostra/Artigos/ SAUDE\%20E\%20BIOLOGICAS/DESCARTE $\% 20$ DE $\% 20 M E D I C A M E N T O S \% 20 V E N C I D O S \% 200 U \% 20$ N\%C3\%83O \%20UTILIZADOS\%20RISCOS\%20E $\% 20$ AVAN\%C3\%87OS\%20RECENTES.pdf.

SANDESON, H.; BRAIN, R. A.; JOHNSON, D. J.; WILSON, C. J.; SOLOMON, K. R. Toxicity classification and e valuation of four Pharmaceuticols classes: antibiotics, antineoplastic, cardiovascular and sex hormones. Toxicology, v. 203, n. 1/3, p. 27-40, 2004.

SIQUEIRA, K. M.; BRANDÃO, J. R.; LIMA, H. F.; GARCIA, A. C. A.; GRATONE, F. M.; BRASILEIRO, M. S. E. Perfil das intoxicações exógenas infantis atendidas em um hospital especializado da rede pública de GoiâniaGO. Rev. Eletr. Enf., v. 10, n. 3, p. 662-72, 2008.

TESSARO, P. R.; ZANCANARO, V. Recolhimento e descarte dos medicamentos das farmácias caseiras no município de caçador - SC. Saúde Meio Ambiente, v. 2, n. 1, p. 118-128, 2013.

TESSEROLLI, D. A.; NEUBERN, F. J.; MACÊDO, J. T.; NAIDE, S. S. Descarte de medicamentos: a visão da comunidade acadêmica e das farmácias. Revista Ciências do Ambiente On-Line, v. 9, n. 2, p. 96 -101, 2013.

VARGAS, J. A. Descarte de medicamentos: desafios e possibilidades na implementação da logística reversa de medicamentos no município de vitória - ES. 2014. 110 f. Dissertação (Mestrado em políticas públicas e desenvolvimento local), ESCSM, 2014.

XU, N.; XU, Y. F.; XU, S.; LI, J.; TAO, H. C. Removal of estrogens in municipal waste water treatment plants: A 
Chinese perspective. Environmental Pollution, v. 165, p. 215- 224, 2012.

ZAPPAROLI, D.; CAMARA, M. R. G.; BECK, C. Medidas Mitigadoras para a Indústria de Fármacos Comarca de Londrina - PR, Brasil: Impacto Ambiental do Despejo de Resíduos em Corpos Hídricos. In: 3rd International Workshop | Advances in Cleaner Production, 2011.

ZHANG, X.; OAKES, K. D.; CUI, S.; BRAGG, L.; SERVOS, M. R.; PAWLISZYN, J. Tissue-specific in vivo bioconcentration of pharmaceuticals in rainbow trout (Oncorhynchusmykiss) using space-resolved solidphase microextraction. Environ. Sci. Technol, v. 44, n. 9, p. 3417-3422, 2010.

ZUCCATO, E. et al. Pharmaceuticals in the environment in Italy: causes, occurrence, effects and control. Environ. Sci. Pollut. Res. Int., v. 13, n. 1, p. 15-21, 2006. 